\title{
MELHORIA NO DESEMPENHO DE MOINHO DE TRIGO ATRAVÉS DO SISTEMA DE CHÃO-DE-FÁBRICA
}

\section{WHEAT MILLING PERFORMANCE IMPROVEMENT USING A "SHOP- FLOOR SYSTEM"}

\author{
Maico Jeferson de Oliveira ${ }^{1}$ Kazuo Hatakeyama ${ }^{2}$ \\ ${ }^{1}$ Federal Technological University of Paraná - UTFPR - Ponta Grossa - Brasil \\ maico@agraria.com.br \\ ${ }^{2}$ Federal Technological University of Paraná - UTFPR - Ponta Grossa - Brasil \\ hatakeyama@utfpr.edu.br
}

\begin{abstract}
Resumo
Neste artigo é apresentada uma proposta de sistema de chão-de-fábrica para utização na agroindústria de moagem de trigo. A modelagem foi realizada através do levantamento das necessidades dos moinhos e da pesquisa bibliográfica. A ênfase consiste em melhorar o desempenho dos moinhos. A proposta foi simulada em um dos moinhos e os resultados são avaliados neste artigo .
\end{abstract}

Palavras-chave: moinho de trigo, controle, eficiência.

\section{Introdução}

A agroindústria de moagem de trigo carece de estudos com objetivo de inserir melhorias na gestão do sistema produtivo. O controle Estatal no setor que perdurou por mais de três décadas "isolou" os moinhos com reflexos negativos ao desenvolvimento competitivo. A capacidade atual de moagem instalada no país é maior do que a demanda por farinha de trigo e a importação proveniente da Argentina agrava ainda mais a competitividade da indústria nacional.

Os sistemas de chão-de-fábrica podem proporcionar maior competitividade para a agroindústria de moagem de trigo através de melhorias no uso dos recursos de produção e na qualidade e precisão das atividades de planejamento da produção.

Este artigo apresenta uma proposta de sistema de chão-de-fábrica que poderá ser utilizado pelos moinhos para obtenção de melhorias. Para o desenvolvimento da modelagem foram utilizadas informações sobre as necessidades dos moinhos, obtidas através de uma pesquisa exploratória com quatorze moinhos, mediante a utilização de questionários. As necessidades dos moinhos juntamente com a pesquisa bibliográfica formaram a base para o desenvolvimento da proposta que foi simulada 
em um dos moinhos para a sua avaliação.

\section{Sistema de chão-de-fábrica}

Em geral duas abordagens são aplicadas para o controle de chão-de-fábrica. Uma é fundamentada no monitoramento de ordens de produção que transitam pelo sistema, o Shop-Floor Control (SFC), utilizado nos sistemas MRP II (Manufacturing Resouce Planning). A outra é baseada no monitoramento das taxas de fluxo na fábrica, que é amplamente empregada nos sistemas Just in time (JIT) (VOLLMANN et al. 2006).

O SFC comporta operações de produção em lote, as ordens são acompanhadas pela fábrica e os desvios são comunicados através de relatórios de fábrica e de análise de entrada/saída fornecidos pelo sistema (CORREIA; GIANESI; CAON, 2001).

$\mathrm{Na}$ abordagem baseada no controle do fluxo a ênfase está na taxa de fluxo, suportando sistemas com variedade limitada. A abordagem JIT é baseada em tempo de fluxo mínimo para o produto inteiro. Há pouca ou nenhuma competição por capacidade e o acompanhamento da ordem é inexistente, uma vez que o material é movimentado rapidamente pelo sistema. (MAYER, 1992; MOREIRA, 1990; VOLLMANN et. al. 2006).

DIAS (2004) discorre em seu trabalho sobre as atividades de chão-de-fábrica e propõe um sistema de controle enfatizando a importância dos indicadores de produção. FAVORETO e VIANA (2006) também chamam a atenção para a utilização de indicadores na operação.

O feedback rápido das informações é uma variável importante nos sistemas de chão-defábrica (TUBINO, 2000). JUNQUEIRA (2003) estuda a coleta de dados através de sistemas supervirórios com o objetivo de uso nas atividades de programação e controle da produção, proporcionando maior integração. FAVORETTO (2001) propõe uma modelagem de PCP que recebe os dados coletados automaticamente da produção. MURBACK, PAIVA e CARVALHO (2006) também analisam indicadores coletados automaticamente do chão-de-fábrica e constatam a ineficácia na coleta manual de dados, realizada antes da utilização do sistema de controle automatizado. FORTULAN e FILHO (2005) realizaram aplicação do Business Intelligence (BI) para transformar dados de chão-de-fábrica em informações para a tomada de decisão. STRUMIELLO (1999, p.133) em sua pesquisa relata a dificuldade encontrada na coleta de dados de chão-de-fábrica feita manualmente:

O grande problema encontrado é que os controles não são corretamente executados, e, além disso, não há registros dos mesmos, dificultando o estabelecimento de um padrão, que identifique os desvios normais e anormais, prejudicando também a detecção de desperdícios no processo. 


\section{Proposta de sistema de chão-de-fábrica}

O perfil das práticas utilizadas pelos moinhos no de chão-de-fábrica é apresentado na Tabela 1.

TABELA 1 - Métodos de controle utilizados nos moinhos

\begin{tabular}{|c|c|c|c|}
\hline Método de monitoramento & $\begin{array}{l}\text { Freqüência } \\
\text { absoluta }\end{array}$ & $\begin{array}{l}\text { Freqüência } \\
\text { absoluta } \\
\text { acumulada }\end{array}$ & $\begin{array}{c}\text { Freqüência } \\
\text { Relativa }\end{array}$ \\
\hline Extração dos moinhos & 8 & 8 & $15 \%$ \\
\hline Monitoramento de ordem de produção & 7 & 15 & $13 \%$ \\
\hline Industrialização do período & 6 & 21 & $12 \%$ \\
\hline $\begin{array}{l}\text { Comparação entre } \\
\text { planejado }\end{array}$ & 6 & 27 & $12 \%$ \\
\hline Paradas de produção & 6 & 33 & $12 \%$ \\
\hline Fluxo de produção nas linhas de envase & 5 & 38 & $10 \%$ \\
\hline Fluxo de trigo na moagem & 4 & 42 & $8 \%$ \\
\hline Fluxo de trigo na preparação & 3 & 45 & $6 \%$ \\
\hline Produtividade ou eficiência na moagem & 3 & 48 & $6 \%$ \\
\hline Outros métodos & 3 & 51 & $6 \%$ \\
\hline $\begin{array}{l}\text { Produtividade ou eficiência nas linhas } \\
\text { de envase }\end{array}$ & 1 & 52 & $2 \%$ \\
\hline $\begin{array}{l}\text { Produtividade ou eficiência } \\
\text { preparação de trigo }\end{array}$ & 0 & 52 & $0 \%$ \\
\hline$\sum$ & 52 & & $100 \%$ \\
\hline
\end{tabular}

Fonte: Coleta de campo.

O foco dos moinhos pesquisados é a extração, um importante indicador que representa a eficiência do processo na obtenção da farinha de trigo. Porém a extração não expressa integralmente a eficiência do sistema de produção. A ênfase no monitoramento de ordens de produção chama a atenção, pois o processo de moagem é contínuo, sugerindo uma abordagem baseada em taxa de fluxo.

Os períodos de feedback das informações da produção são demonstrados na figura 1. 
FIGURA 1 - Período de verificação do monitoramento da produção nos moinhos

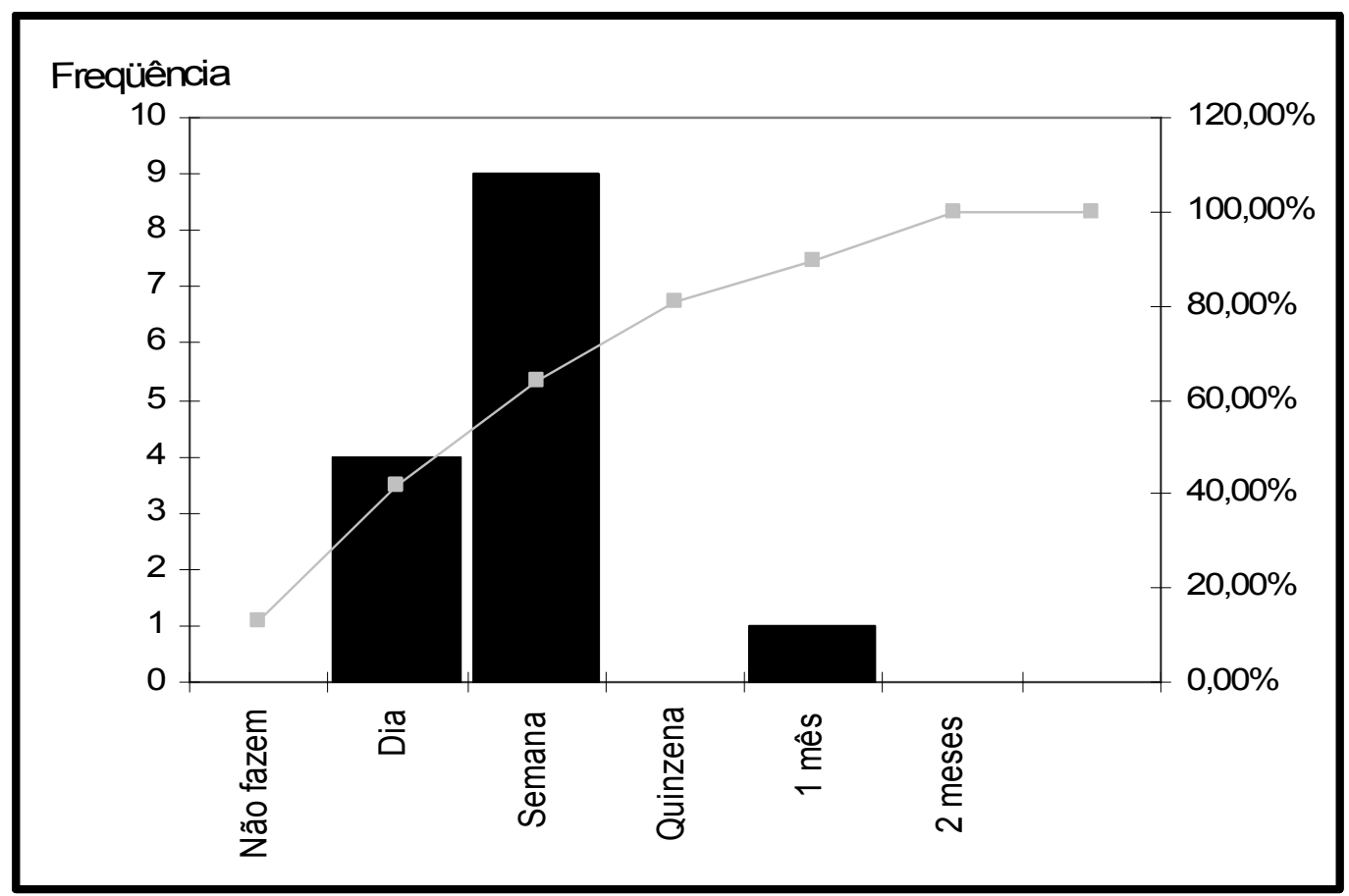

Fonte: Coleta de campo

O padrão observado de intervalo para análise e correção dos desvios é longo, contrapondose ao elevado fluxo de material que transita pelo sistema, isto poderá comprometer a confiabilidade dos planos de PCP.

A proporção de controle adotado nas etapas de produção, segundo os resultados da pesquisa de campo é ilustrado na Figura 2.

FIGURA 2 - Proporão de controle aplicado nas etapas de produção

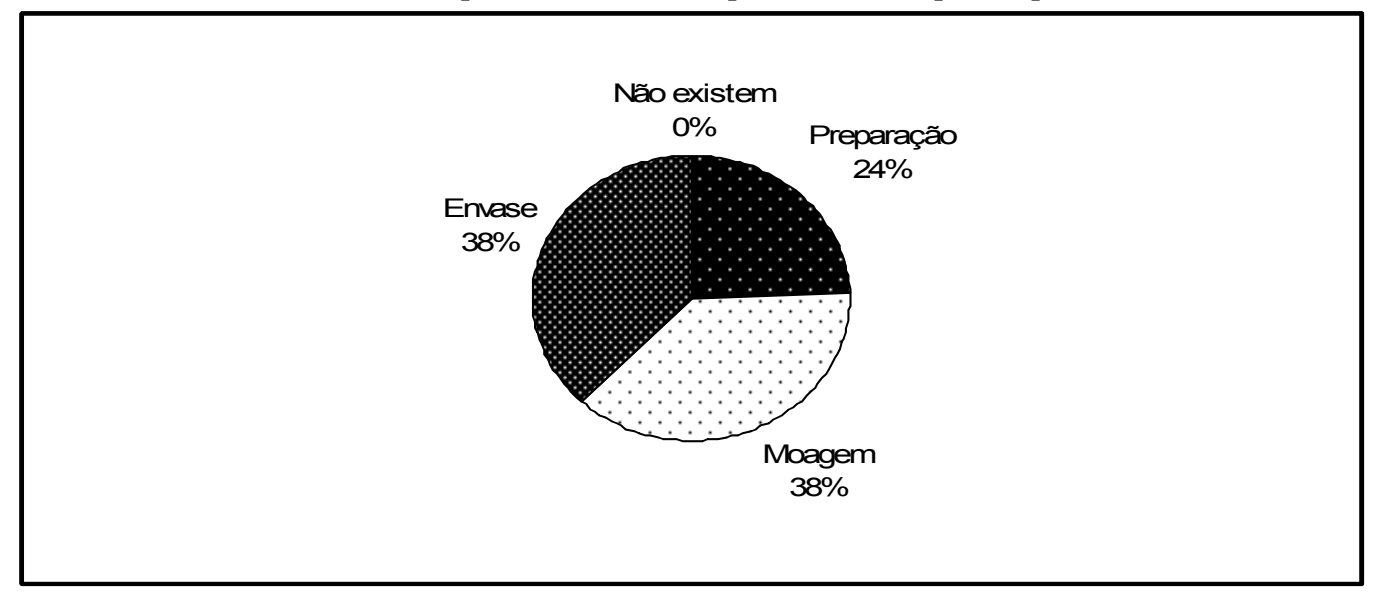

Fonte: Coleta de campo

Há um equilíbrio na proporção de controle empregado nas etapas, que pode representar um alerta. A etapa de moagem é o recurso "gargalo" de todo o sistema, pois a preparação de trigo 
possui capacidade maior em relação à moagem para garantir o tempo de repouso necessário ao processo. As linhas de envase também apresentam capacidade elevada para assegurar o mix de produtos, cuja racionalização da utilização deve ser considerada.

O controle de chão-de-fábrica pode representar uma atividade que demanda grande capacidade de processamento, isto ocorre quando a ênfase está no acompanhamento da movimentação de uma ordem de produção pelo sistema produtivo. Os sistemas de chã-de-fábrica que suportam a abordagem SFC são exemplos de sistemas que demandam alta capacidade de processamento. No ambiente JIT a redução de lead time e estoques tornaram o fluxo tão rápido que o acompanhamento de ordens específicas foi substituído pela taxa de fluxo. Nas indústrias de produção contínua a alta velocidade do fluxo de materiais é condicionada pelas características operacionais do ambiente produtivo.

A proposta de sistema de chão-de-fábrica para moinho de trigo é baseada no monitoramento de fluxo. O foco é o controle, pressupondo o sequênciamento da produção numa etapa anterior de scheduling. Uma premissa é o processamento rápido, pois o fluxo de material é veloz, potencializando a ocorrência de grandes desvios.

A facilidade da coleta de dados na produção é simplificada pela redução de eventos que deverão ser apontados, sendo a freqüência dos apontamentos uma função da necessidade de cada moinho em obter os resultados de um turno, dia ou tipo de produto. Uma visão global da proposta de sistema de chão-de-fábrica para moinho de trigo é ilustrada na figura 3.

FIGURA 3 - Proposta de sistema de chão-de-fábrica para moinho de trigo

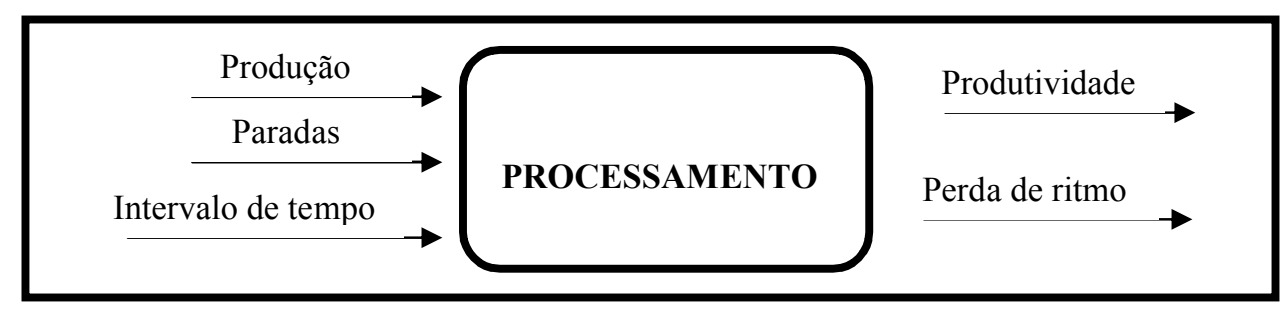

Fonte: Os autores

A modelagem apresenta três variáveis independentes de entrada (inputs) e duas dependentes de saída (outputs). Na tabela 1 é possível constatar que há uma diferença entre os moinhos que utilizam a produtividade nas atividades de controle e os que utilizam as paradas para tal finalidade. Demonstrando que as paradas e a produtividade são independentes entre si em alguns casos, incorrendo no risco de obter alta produtividade mesmo que ocorra baixa quantidade produzida.

As paradas de produção podem ser obtidas diretamente dos sistemas supervisórios, reduzindo as necessidades de apontamentos. A mesma abordagem poderá ser aplicada na coleta da 
quantidade produzida e do intervalo de tempo de produção. A adoção de um sistema de códigos de paradas poderá facilitar os apontamentos.

O processamento não demanda grande recurso computacional, podendo ser realizado por meio de planilhas de cálculos, à custa da menor acurácia e rastreabilidade das informações. A estrutura ideal seria aquela em que os dados são coletados diretamente dos sistemas supervisórios e processados em um software modelado. O processamento ocorre de acordo com as equações:

$$
\mathbf{\rho}=\mathbf{H p}-(\mathbf{Q} / \mathbf{C P N})
$$

Onde:

a) $\rho$ - Perda de ritmo em horas;

b) $\mathrm{Hp}$ - Horas produtivas;

c) Q - Quantidade produzida em toneladas durante as horas produtivas;

d) CPN - Capacidade nominal da linha de moagem em toneladas por hora.

$$
\mathbf{P}=\mathbf{1}-(\boldsymbol{\rho}+\boldsymbol{\theta}) / \mathbf{H t} \quad \text { (eq. } 2)
$$

Onde:

a) P - Produtividade;

b) $\theta$ - Paradas da produção não programadas;

c) $\mathrm{Ht}$ - Horas totais do período.

Propõe-se o monitoramento na etapa de moagem. Esta etapa constitui-se no recurso crítico de capacidade de todo o sistema, sua eficiência estará "puxando" as etapas antecedentes e ao mesmo tempo "empurrando" as etapas posteriores, garantindo a eficiência de todo o sistema. Numa segunda abordagem as etapas que representam "gargalos" em relação à moagem podem ser monitoradas, porém isto vai depender de cada situação, pois as etapas anteriores têm suas capacidades desbalanceadas para obtenção do tempo de repouso antes da moagem. As etapas posteriores também apresentam capacidades maiores para garantir o mix de produção final.

A produtividade, que será a base para as atividades de programação, é o feedback do módulo ao sistema geral de PCP. A comparação entre a produtividade padrão e a real fornecerá evidências indiretas do cumprimento da programação baseada em taxas de fluxos, ao mesmo tempo em que as paradas de produção e a perda de ritmo estarão representando o nível de eficiência do sistema produtivo.

\section{Simulação da proposta de chão-de-fábrica}

A simulação da proposta de sistema de chão-de-fábrica foi realizada em um moinho de trigo de porte médio para os padrões nacionais, composto por dois diagramas de moagem. Os dados resultantes da aplicação da modelagem foram coletados durante onze meses de funcionamento do 
sistema produtivo. Os resultados das paradas e das perdas de ritmo fornecidos pelo sistema de chãode-fábrica são ilustrados graficamente na figura 4.

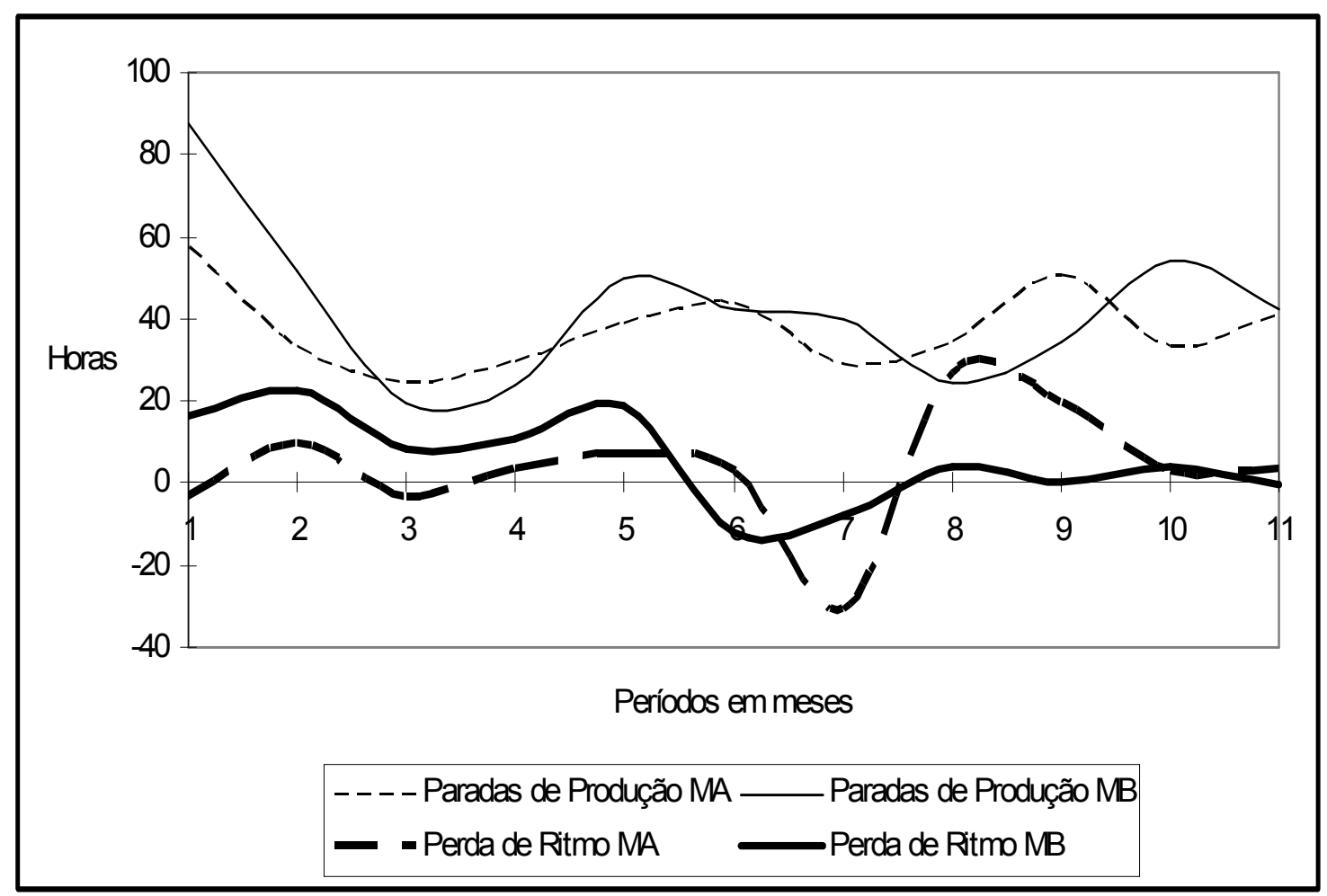

FIGURA 4 - Desempenho em perda de ritmo e paradas não programadas de duas linhas de moagem de trigo Fonte: Simulação da proposta pelos autores

Ao longo do funcionamento dos moinhos verificou-se a ocorrência de ciclos a cada três períodos. Os ciclos coincidiram com a freqüência das manutenções preventivas gerais da fábrica, que são realizadas a cada três meses em conjunto com as tarefas de higienização dos equipamentos.

Nos períodos que antecederam as manutenções verificaram-se os pontos máximos, indicando a eficácia da manutenção sobre os indicadores de desempenho das linhas de produção, uma vez que logo após as manutenções os valores apresentados são os menores. Desta forma o sistema de chão-de-fábrica pode ser utilizado no apoio às decisões de manutenção, abrangendo à eficácia das manutenções e a freqüência. No que diz respeito à freqüência das manutenções, conforme indicam os ciclos da figura 4, uma das maneiras de melhorar o desempenho do sistema produtivo seria o aumento da freqüência das manutenções, desta forma estes dados poderiam ser usados na gestão da manutenção. Uma análise mais apurada dos dados pode indicar alguns equipamentos fundamentais que causam impacto no desempenho do sistema produtivo, e com base em informações da gestão da manutenção algumas ações de melhorias mais específicas podem ser motivadas. 
A produtividade fornecida pelo sistema de chão-de-fábrica nas linhas de moagem "MA" e "MB" ao longo dos onze meses é demonstrada na figura 5.

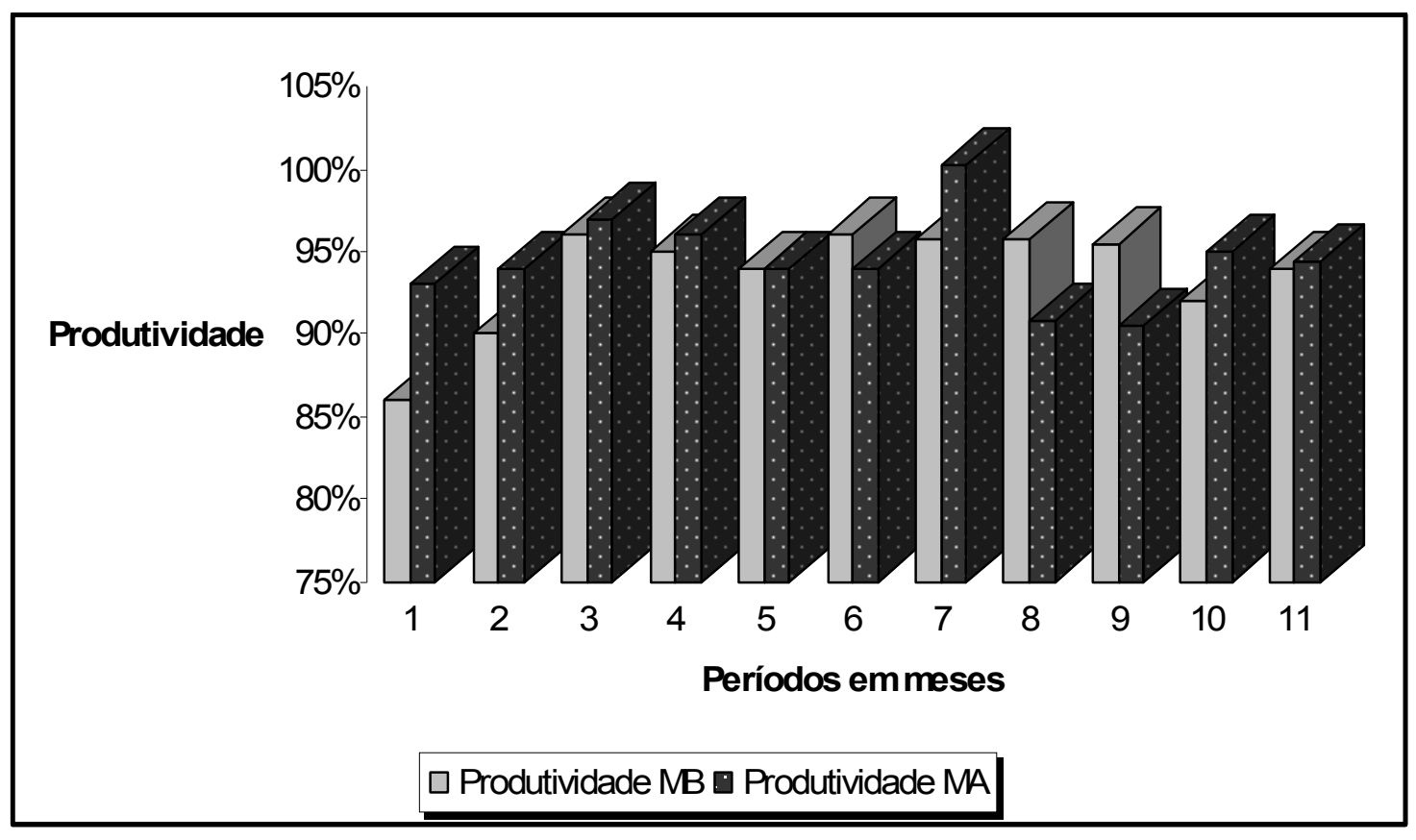

FIGURA 5 - Produtividade nas linhas de moagem MA e MB Fonte: Simulação da proposta pelos autores

Verifica-se que a produtividade é menor nos períodos iniciais logo após a introdução do sistema. Antes da simulação a produtividade era utilizada para monitorar o desempenho das linhas de produção, porém a metodologia de obtenção da produtividade resultava em índices elevados, mesmo com o output reduzido. Desta forma a produtividade não garantia a quantidade produzida, comprometendo os planos de PCP. O mesmo fenômeno pode ocorrer nos moinhos da tabela 1 que não consideram as paradas e a produtividade em conjunto. A tabela 2 faz uma estatística dos resultados obtidos do sistema de chão-de-fábrica.

TABELA 2 - Estatística dos dados do sistema de chão-de-fábrica

\begin{tabular}{|l|c|}
\hline \multicolumn{1}{|c|}{ Indicadores } & Resultado \\
\hline Correlação entre a perda de ritmo e a produtividade na linha MA & $-0,90$ \\
\hline Correlação entre as paradas não programadas e a produtividade na linha MA & $-0,64$ \\
\hline Produtividade média da linha MA & $94 \%$ \\
\hline Correlação entre a perda de ritmo e a produtividade na linha MB & $-0,60$ \\
\hline Correlação entre as paradas não programadas produtividade na linha MB & $-0,89$ \\
\hline Produtividade média da linha MB & $94 \%$ \\
\hline
\end{tabular}

Fonte: Simulação da proposta pelos autores

É possível verificar que a produtividade da linha "MA" está correlacionada negativamente à perda de ritmo. Desta forma incrementos na produtividade podem ser obtidos reduzindo a perda de ritmo nesta linha. Sob o aspecto operacional a perda de ritmo pode estar relacionada às falhas operacionais e deficiências na preparação do trigo para a moagem ou no sistema de transporte pneumático da linha de produção. 
Por outro lado a produtividade na linha "MB" está correlacionada negativamente às paradas não programadas, sugerindo que o incremento de melhorias na produtividade pode ocorrer pela redução de falhas nos equipamentos.

A estatística das informações provenientes do sistema de chão-de-fábrica forneceu evidências de que ações diferenciadas deveriam ser motivadas para cada linha de produção, visando o mesmo objetivo, o aumento da produtividade, que tem como conseqüência a eficiência do processo e a confiabilidade dos planos de PCP. Para ambas as linhas de produção uma ação comum relacionada à gestão da manutenção poderia ser realizada para melhorar a produtividade. A modelagem revelou necessidades específicas para cada linha, o que contribui para a obtenção de respostas rápidas quando ocorrem desvios.

\section{Considerações finais}

O sistema de chão-de-fábrica proposto neste trabalho poderá ser utilizado em conjunto com o indicador de extração, amplamente utilizado nos moinhos, para o monitoramento integral do sistema de produção. A produtividade não visa a substituição do indicador de extração, visto que ambos se complementam.

A proposta cumpre sua finalidade de proporcionar respostas rápidas, característica evidenciada em vários trabalhos sobre o tema. Dois fatores são responsáveis pelas respostas rápidas proporcionadas pelo sistema de chão-de-fábrica: a redução das entradas e saídas gerando somente informações verdadeiramente úteis ao sistema produtivo; e, a possibilidade de coleta de dados diretamente dos sistemas supervisórios dos moinhos.

A pesquisa exploratória revelou uma deficiência em grande parte dos moinhos ao adotar a estratégia de monitoramento da movimentação de ordens de produção específicas, já que a produção nos moinhos é de processamento contínuo. Porém isto não deverá ser entendido como uma necessidade de banir o uso de ordens de fabricação nos moinhos, privando-os de usufruir de benefícios como rastreabilidade e a acurácia no levantamento dos custos diretos de produção obtidos através da ordem de produção. Pretende-se aqui motivar uma análise crítica quanto ao trade-off proporcionado pelo esforço desprendido para monitorar uma ordem em seu deslocamento pelo sistema de produção dos moinhos.

O apontamento da produção é a base para a atualização do estoque físico. Pretende-se que a informação dos estoques disponíveis e da produtividade sirvam de entradas para o Master Planning Scheduling (MPS) onde juntamente com as informações de produções já confirmadas irão formar a base para a realização do MPS. A produtividade informada pelo sistema de chão-de-fábrica poderá ser utilizada para o carregamento finito dos moinhos, podendo ainda a utilização de médias móveis da produtividade para validar a capacidade. 
Por fim, é importante destacar o enfoque da proposta no monitoramento da eficiência de utilização dos recursos de produção. A modelagem mostrou-se sensível às necessidades de reposta ao sistema de PCP (produção x produtividade) e aos pontos de correção para a obtenção de melhorias.

\begin{abstract}
This manuscript presented a "shop-floor system" proposal that can be used in a wheat milling facility. The system was based on a model that considers wheat milling needs and literature review. The main objective of this model was to improve the wheat milling performance. The proposed model was applied in a mill and the results are evaluated in this paper in order to submit this system.
\end{abstract}

Key-words: wheat milling, control, performance.

\title{
Referências
}

CORREIA, H. L; GIANESI; CAON. Planejamento Programação e Controle da Produção. 2. ed. São Paulo: Atlas, 2001.

DIAS, S. L. V. Proposta de uma Sistemática de Controle de Chão de Fábrica no Curtissímo Prazo Apartir do Indice de Eficiência Global, o IEG. In: ENCONTRO NACIONAL DE ENGENHARIA DE PRODUÇÃO, 24. , 2004, Anais... Florianópolis: UFSC SC, 2004, 1CD.

FAvoretto, F. Uma Contribuição ao Processo de Gestão da Produção Pelo Uso da Coleta Automática de Dados de Chão de Fábrica. 2001, 222 f. Tese de Doutorado (Doutorado em Engenharia Mecânica) - Escola de Engenharia de São Carlos da Universidade de São Paulo, São Carlos, 2001.

FAVOretto, F; VIEIRA, G. E. Indicadores de Controle da Produção para Suporte da Estratégia de Manufatura. SIMPÓSIO DE ENGENHARIA DE PRODUÇÃO, 13. , 2006, Anais... Bauru: UNESP SP, 2006.

FORTUlan, R. M; FILHO, E.V.G. Uma Proposta de Aplicação de Business Intelligence no Chão-de-fábrica. Gestão \& Produção. Vol. 12, nº. 1, jan-abr. 2005, p.55-66.

cross ${ }^{\text {ref }}$

JUNQUEIRA, G. S. Análise das Possibilidades de Utilização de Sistemas Supervirórios no Planejamento e Controle da Produção. 2003, 131 f. Dissertação de Mestrado (Mestrado em Engenharia de Produção) - Escola de Engenharia de São Carlos da Universidade de São Paulo, São Carlos, 2003.

MAYER, R. R. Administração da Produção. São Paulo: Atlas, 1992.

MOREIRA, D. A. Administração da produção e Operações. São Paulo: Pioneira, 2004.

MURBACK, G. R; PAIVA, M. C; CARVALHO, M. F. H. A Influência da Visibilidade da Informação no Chão de Fábrica no Desempenho da Manufatura. SIMPÓSIO DE ENGENHARIA DE PRODUÇÃO, 13. , 2006, Anais... Bauru: UNESP SP, 2006.

STRUMiello, L. D. P. Proposta de Planejamento e Controle da Produção e Custos para Pequenas Empresas do Vestuário. 1999, 161 f. Dissertação (Mestrado em Engenharia de Produção) - Programa de Pós-graduação em Engenharia de Produção, Universidade Federal de Santa Catarina, Florianópolis, 1999.

TUBINO, D. F. Manual de Planejamento e Controle da Produção. 2. ed. São Paulo: Atlas, 2000.

VOllmann, T. E. et. al. Sistemas de Planejamento \& Controle da Produção Para o Gerenciamento da Cadeia de Suprimentos. 5. ed. São Paulo: Bookman, 2006. 
Dados completos dos autores:

\section{Maico Jeferson de Oliveira}

Cooperativa Agraria Mista Entre Rios ltda.

Departamento: Moinho de Trigo

Supervisor

Rua Domingos Marcondes, 577, Santana, Guarapuava, Paraná, Brasil. CEP 85070020

4236225369

maico@agraria.com.br

\section{Kazuo Hatakeyama}

Universidade Tecnológica Federal do Paraná -UTFPR

Departamento de Pós-Graduação em Engenharia de Produção - PPGEP

Professor Adjunto

Avenida Monteiro Lobato km 04, Jardim Pitangui

CEP: 84016-210 - Ponta Grossa, PR - Brasil - Caixa-Postal: 20

(42) 32204878

Recebido para publicação em: 10/07/07

Aceito para publicação em: 07/08/07 